Article

\title{
SAXS Studies of the Endoglucanase Cel12A from Gloeophyllum trabeum Show Its Monomeric Structure and Reveal the Influence of Temperature on the Structural Stability of the Enzyme
}

\section{Lis S. Miotto ${ }^{1}$, Caio V. dos Reis ${ }^{1}$, Mario de Oliveira Neto ${ }^{2}$ and Igor Polikarpov ${ }^{1}{ }^{*} *$}

1 Grupo de Biotecnologia Molecular, Instituto de Física de São Carlos, Universidade de São Paulo, Av. Trabalhador São-Carlense 400, Arnold Schimidt, São Carlos, SP 13566-590, Brazil;

E-Mails: lismiotto@yahoo.com.br (L.S.M.); caio.reis@usp.br (C.V.R.)

Departamento de Física e Biofísica, Instituto de Biociências de Botucatu,

Universidade Estadual Paulista, Distrito de Rubião Júnior, S/N, Botucatu, SP 18618-970, Brazil;

E-Mail: mario.neto@ibb.unesp.br

* Author to whom correspondence should be addressed; E-Mail: ipolikarpov@ifsc.usp.br;

Tel.: +55-16-3373-9874; Fax: +55-16-3373-9881.

Received: 25 April 2014; in revised form: 10 June 2014 / Accepted: 24 June 2014 /

Published: 17 July 2014

\begin{abstract}
Endoglucanases are key enzymes applied to the conversion of biomass aiming for second generation biofuel production. In the present study we obtained the small angle X-ray scattering (SAXS) structure of the G. trabeum endo-1,4- $\beta$-glucanase Cel12A and investigated the influence of an important parameter, temperature, on both secondary and tertiary structure of the enzyme and its activity. The $\mathrm{CD}$ analysis for GtCel12A revealed that changes in the $\mathrm{CD}$ spectra starts at $55{ }^{\circ} \mathrm{C}$ and the $T_{\mathrm{m}}$ calculated from the experimental $\mathrm{CD}$ sigmoid curve using the Boltzmann function was $60.2 \pm 0.6{ }^{\circ} \mathrm{C}$. SAXS data showed that GtCel12A forms monomers in solution and has an elongated form with a maximum diameter of $60 \pm 5 \AA$ and a gyration radius of $19.4 \pm 0.1 \AA$ as calculated from the distance distribution function. Kratky analysis revealed that $60{ }^{\circ} \mathrm{C}$ is the critical temperature above which we observed clear indications of denaturation. Our results showed the influence of temperature on the stability and activity of enzymes and revealed novel structural features of GtCel12A.
\end{abstract}


Keywords: endoglucanase; Gloeophyllum trabeum; biophysics; circular dichroism; small angle X-ray scattering (SAXS); Kratky analysis

\section{Introduction}

Brown rot fungi are the most important agents involved in the degradation of wood products, which occurs both by enzymatic and non-enzymatic mechanisms. During this process, these fungi actively metabolize the carbohydrate portion of wood by breaking down cellulose and hemicellulose, leaving the lignin portion intact [1]. This feature holds an interesting property for industrial bioconversion [2].

Gloeophyllum trabeum is a representative specimen of this group and causes a typical brown rot, following peculiar pattern of wood decay during which wood cell wall components are oxidatively broken down, causing a rapid loss of wood strength [1]. The depolymerization of cellulose and hemicelluloses from the plant cell wall by G. trabeum occurs through the generation of hydroxyl radicals via the Fenton reaction $\left(\mathrm{H}_{2} \mathrm{O}_{2}+\mathrm{Fe}^{2+}\right)$ assisted by hydroquinones, and secretion of low molecular weight $\left(M_{\mathrm{w}}\right)$ peptides. The hydroxyl radicals in turn become able to depolymerize plant cell wall polysaccharides due to their strong oxidizing capability [3].

Endoglucanases (EGs) also known as endo-1-4- $\beta$-glucanases are a type of cellulase that attack initially and randomly multiple internal sites of the amorphous regions of the cellulose fiber. These provide sites for subsequent attack by cellobiohydrolases [4]. EGs catalyze the hydrolysis of internal connections, $\beta-1,4-\mathrm{D}-\mathrm{glucosidic}$ linkages in cellulose, and are also capable of hydrolyzing $\beta-1,4-D-g l u c a n s$ also containing $\beta-1,3$ bonds [5,6]. EGs and other glycoside hydrolases and transglucosidases have been classified into glycoside hydrolases (GHs) families according to their substrate specificity and molecular mechanism in a Carbohydrate-Active EnZymes (CAZy) database $[7,8]$.

The glycoside hydrolase family 12 in which GtCel12A is included can be divided into subfamilies and very few examples of GH12 family members have carbohydrate-binding modules (CBMs). GH12 members are found in a wide range of organisms including numerous extremophile species such as the archeon Pyrococcus furiosus [9], the hyperthermophilic bacteria Thermotoga neapolitana [10], the thermophilic eubacteria Rhodothermus marinus [11] and the thermotolerant acidophilic bacteria Acidothermus cellulolyticus [12].

In this study, we determined the small angle X-ray scattering (SAXS) structure and investigated the effect of temperature on the structural stability, compactness and activity of the endo-1,4- $\beta$-glucanase Cel12A from G. trabeum, a GH12 member, following different biophysical and biochemical approaches.

\section{Results and Discussion}

\subsection{GtCel12A Is One of the Most Thermal Stable among Other Characterized Cel12A}

Analysis of GtCel12A by circular dichroism (CD) revealed a predominant $\beta$-strand structure, which corroborated the typical $\beta$-sheets based fold commonly found in GH12 family members. 
Deconvolution of the GtCel12A spectrum showed that its secondary structure consists of $35.1 \%$ $\beta$-strands, $19.3 \% \beta$-turns, $6.9 \%$ helices and $38.7 \%$ disordered residues.

Thermal denaturation assay revealed that changes in the $\mathrm{CD}$ spectra were observed above $55{ }^{\circ} \mathrm{C}$, suggesting a perturbation in the secondary structure for GtCel12A (Figure 1). Boltzmann fit to the experimental curve on $\mathrm{pH} 3.0$ revealed an apparent thermal midpoint $\left(T_{\mathrm{m}}\right)$ of $60.2 \pm 0.6{ }^{\circ} \mathrm{C}$. This value is slightly higher than the $T_{\mathrm{m}}$ values previously reported for other Cel12A from different species, also determined by $\mathrm{CD}$ analysis. The Cel12A from Trichoderma reesei showed a $T_{\mathrm{m}}$ of $54.4{ }^{\circ} \mathrm{C}, 49.2{ }^{\circ} \mathrm{C}$ was observed for the Cel12A from Hypocrea schweinitzii, $58.5{ }^{\circ} \mathrm{C}$ for Trichoderma koningii, $54.1{ }^{\circ} \mathrm{C}$ for Fusarium javanicum and $45.9^{\circ} \mathrm{C}$ for Gliocladium roseum. Only the Cel12A from Streptomyces sp. showed a higher $T_{\mathrm{m}}$ of $65.7^{\circ} \mathrm{C}$ [13]. Moreover, the $T_{\mathrm{m}}$ determined by CD analysis agrees with the $T_{\mathrm{m}}$ equal to $60.4 \pm 0.6{ }^{\circ} \mathrm{C}$ determined for this enzyme at the same $\mathrm{pH}$ using Thermofluor method in our previous experiments (data not shown).

Figure 1. Circular dichroism (CD) spectra for the thermal denaturation assay of GtCel12A. The temperature range is from 10 to $90{ }^{\circ} \mathrm{C}$ varied in steps of $10{ }^{\circ} \mathrm{C}$ in the beginning of the experiment and steps of $5^{\circ} \mathrm{C}$ above $50^{\circ} \mathrm{C}$.

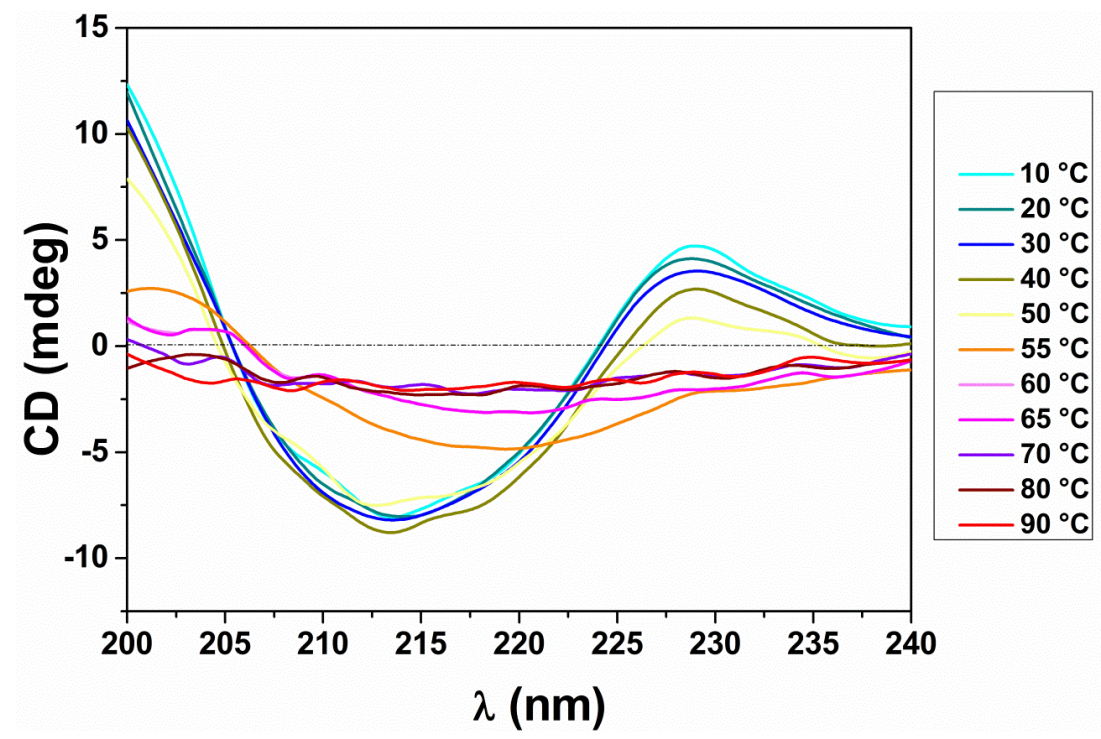

\subsection{GtCel12A Forms Monomers in Solution}

The native polyacrylamide gel electrophoresis (native PAGE) results showed a single band, suggesting sample homogeneity and only one oligomeric form for GtCel12A, which was found suitable for further SAXS analysis.

Aiming to obtain information about size, molecular shape and oligomeric state of the GtCel12A in solution, we submitted the protein to SAXS analysis. Comparative analysis of SAXS scattering curves showed that concentration effects were negligible. With the aim of removing small aggregation effect, the $q_{\text {min }}$ value was reduced to $0.02 \AA^{-1}$. GtCel12A SAXS curve at a concentration of $1 \mathrm{mg} \cdot \mathrm{mL}^{-1}$ is shown in Figure 2a.

The Guinier analysis, in the $q^{2}$-range $R_{\mathrm{g} .} q<1.3$, reproducibly gave estimates of $19.9 \AA$ for the enzyme gyration radius $\left(R_{\mathrm{g}}\right)$. The linearity of the Guinier plot indicated that the preparation was monodisperse. 
Analysis of the distance distribution function $[\mathrm{p}(\mathrm{r})]$ led us to conclude that the protein had an elongated form with a maximum diameter $\left(D_{\max }\right)$ of $60 \pm 5 \AA$ (Figure $2 \mathrm{~b}$ ). The $R_{\mathrm{g}}$ value of $19.4 \pm 0.1 \AA$ calculated from the $\mathrm{p}(\mathrm{r})$ corroborates with the Guinier analysis derived estimate.

The protein $M_{\mathrm{W}}$ estimate of $28.3 \mathrm{kDa}$ was obtained using SAXS MoW web tool [14]. Considering an average $10 \%$ error of the $M_{\mathrm{w}}$ estimates obtained using SAXS MoW [14], this result agrees well with the $M_{\mathrm{w}}$ of GtCel12A monomer computed from its primary sequence $(26.1 \mathrm{kDa})$ by EXPASy Prot Param web tool [15].

Figure 2. GtCel12A analysis by small angle X-ray scattering (SAXS). (a) Experimental scattering curve (open circles) and fits produced both from the homologous xyloglucan-specific endo-beta-1,4-glucanase from Aspergillus aculeatus (AaXEG) structure (PDB ID: 3VL8) (pink line) and from dummy atom model (DAM) (green line). The insert contains Guinier profile to calculate $R_{g} ;(\mathbf{b}) \mathrm{p}(\mathrm{r})$ computed from experimental data.

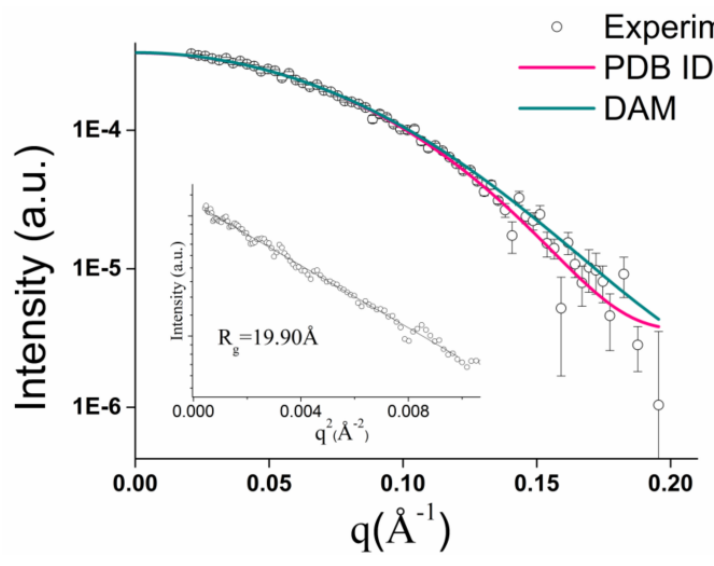

(a)

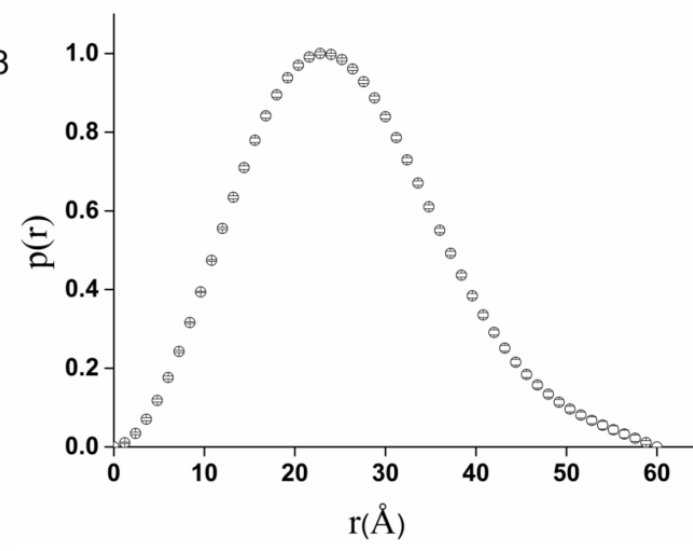

(b)

The DAM for GtCel12A was determined from SAXS data using Gasbor program [16]. Ten independently generated DAMs fitted the experimental SAXS data well. The superposition of DAMs based on normalized spatial discrepancy (NSD) parameter [17] with the homologous AaXEG [18] is shown in Figure 3.

Figure 3. Structural superposition of the crystallographic structure of the homologous AaXEG (pink cartoon) and averaged DAM obtained for GtCel12A by SAXS analysis (green) shown in three different orientations. Right and center models are rotated $90^{\circ}$ around $y$ and $x$ axis, respectively from the left model.

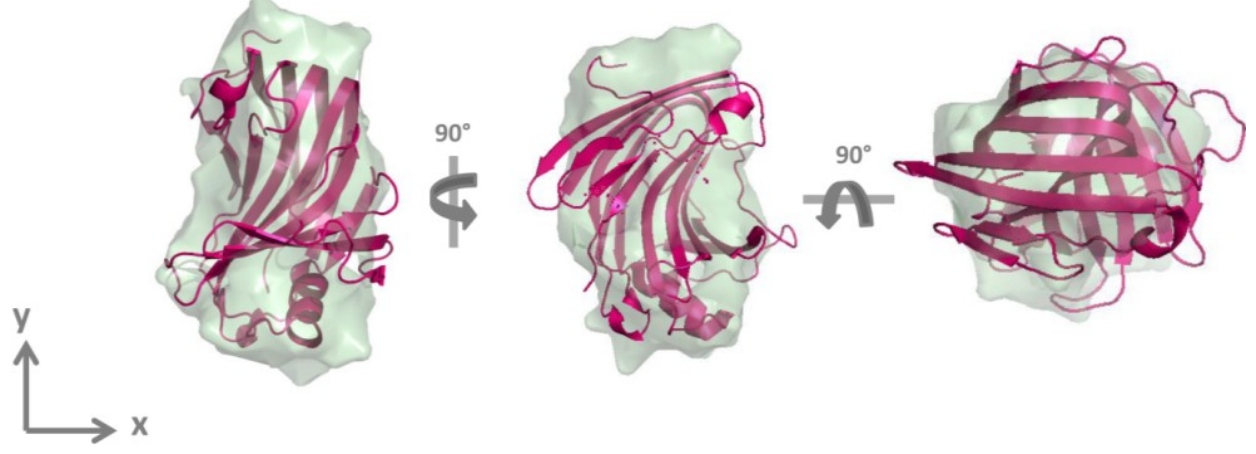


The structural parameters derived from the experimental curve, from the homologous structure and from DAM and the fitting adjustment parameter $\chi$ are shown in Table 1 . The values obtained from these different calculations are similar.

Table 1. GtCel12A and homologous AaXEG structural parameters.

\begin{tabular}{cccc}
\hline Parameters & Experimental & PDB ID: 3VL8 (AaXEG) & DAM \\
\hline$R_{\mathrm{g}}(\AA)$ & $19.4 \pm 0.1$ & 18.25 & 18.57 \\
$D_{\max }(\AA)$ & 60.00 & 53.09 & 56.78 \\
Resolution $(\AA)$ & 32.14 & - & 32.14 \\
SAXS $M_{\mathrm{W}}(\mathrm{kDa})\left(\right.$ theoretical $\left.M_{\mathrm{W}}=26.17\right)$ & 28.30 & - & - \\
$\chi$ & - & 1.40 & 1.47 \\
\hline
\end{tabular}

Detection of conformational switching, destabilization or long-range delocalized flexibility in solution can be investigated by SAXS, typically through qualitative assessments of the scattering data in a Kratky Plot [19,20].

Kratky plot is represented by $\left[I(q) * q^{2} \times q\right]$ curve [20,21], as shown in Figure 4. For small $q$ values and globular enzymes, a parabolic peak is expected to be observed [20,21]. Considering high $q$ values, molecule flexibility can be analyzed: the more ascending the end of the curve is, the more flexible the molecule is [20,21]. The $R_{g}$ and the $M_{\mathrm{w}}$ of GtCel12A remained unaltered up to $55^{\circ} \mathrm{C}$. From $60{ }^{\circ} \mathrm{C}$ on, the parabolic peak shifted to the left, representing an increase in both $R_{g}$ and $M_{\mathrm{w}}$, followed by the abrupt flexibility increase at $65{ }^{\circ} \mathrm{C}$. Therefore, the peak shift indicated that the enzymes in solution were rearranged in a different molecular shape.

Figure 4. Kratky profile $\left[I(q)^{*} q^{2} \times q\right]$ for GtCel12A in $50 \mathrm{mM}$ sodium citrate buffer, $\mathrm{pH} 3.0$ and temperature range from 20 to $65^{\circ} \mathrm{C}$. The presence of a parabolic peak for each temperature reports on the maintenance of enzyme globularity. The progressive peak decrease with no change in horizontal position indicates the loss of tertiary folding. At $60{ }^{\circ} \mathrm{C}$, in addition to flattening of the peak, one can observe a shift to the left in its position. This suggests partial loss of globularity and possible aggregation of the enzyme molecules in solution with simultaneous increase in the $M_{\mathrm{w}}$ of the new ensemble.

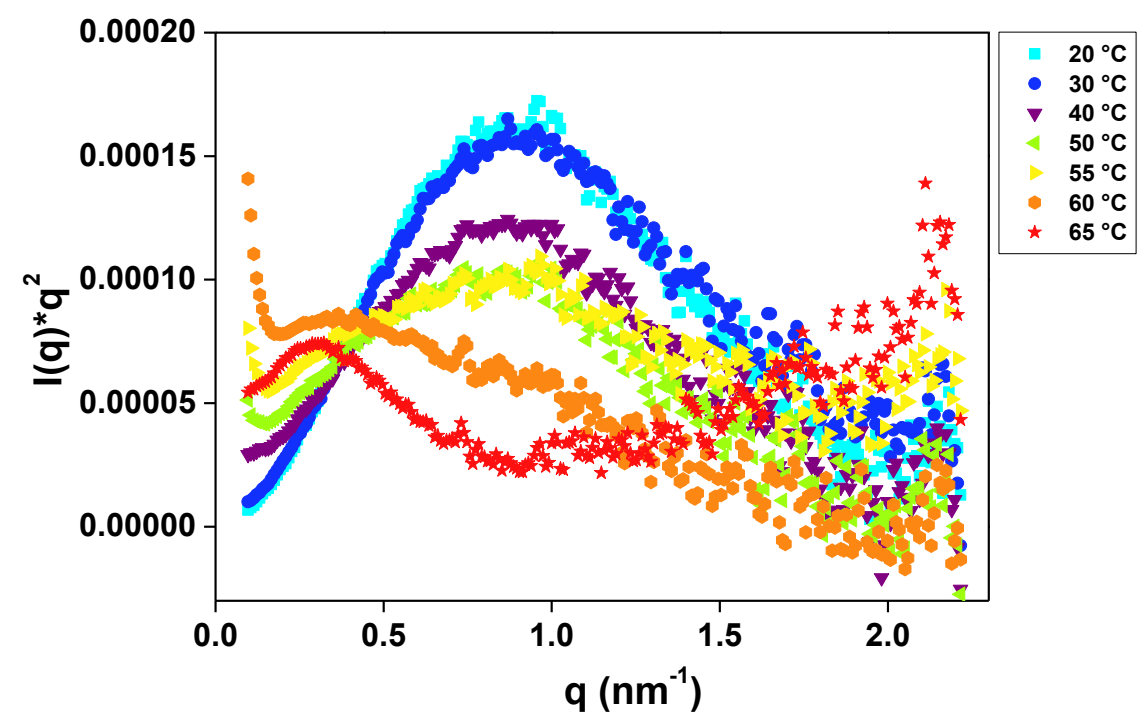


In the independent set of measurements we determined enzymatic activity of GtCel12A using $\beta$-glucan from barley at $1 \%$ as a substrate (to be published elsewhere) which showed that the enzyme's activity began to decrease gradually above $53{ }^{\circ} \mathrm{C}$ and a sudden drop in activity was observed at a critical temperature of $60{ }^{\circ} \mathrm{C}$. At higher temperatures, negligible enzymatic activity was observed, suggesting that most of the molecules were denatured. This result corroborates with SAXS analysis, which showed, by analyzing the molecular shape of the enzyme, that at $60{ }^{\circ} \mathrm{C}$ a clear thermal denaturation occurs, therefore affecting, both the structure stability and activity of GtCel12A.

\section{Experimental Section}

\subsection{Gene Cloning, Recombinant Expression, Protein Production, Purification and} Identity Confirmation

Complementary DNA was prepared by reverse transcription of total RNA isolated from G. trabeum strain ATCC 11539 grown in a medium containing barley and alfalfa $1 \%(\mathrm{w} / \mathrm{v})$ for $32 \mathrm{~h}$ at $34{ }^{\circ} \mathrm{C}$. The gene sequence was selected from GenBank (acession number HQ163778).

Gene cloning into vector ANIp7G, a vector previously constructed from ANIp7 [22] was carried out using the ligation-independent cloning (LIC) method previously described [23]. Recombinant

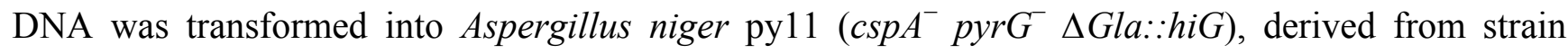
N593 ( $c s p A^{-} p y r G^{-}$), using the protoplast transformation method previously described [24]. Transformants were selected on minimal medium (MM) [25] without uracil and uridine. The ANIp7G vector without the inserted DNA was included as control. Protein production was performed in MM J media, which is similar to MM except that it includes $4 \%(\mathrm{w} / \mathrm{v})$ of maltose as the sole carbon source and the amounts of nitrogen source, salts and trace elements were increased 4-fold. GtCel12A purification was performed in two steps using SP Sepharose, an ion-exchange column, as the first purification step. Fractions were eluted with a linear gradient of $0-1 \mathrm{M}$ of sodium chloride in $50 \mathrm{mM}$ sodium citrate buffer ( $\mathrm{pH}$ 3.0) at a flow rate of $1 \mathrm{~mL} \cdot \mathrm{min}^{-1}$. Samples were analyzed by sodium dodecyl sulfate polyacrylamide gel electrophoresis (SDS-PAGE) and the fractions containing the protein of interest were combined and concentrated using a $10 \mathrm{kDa}$ cut-off Vivaspin ${ }^{\mathrm{TM}}$ (GE Healthcare, Little Chalfont, UK) concentrator. In a second purification step, the concentrated volume was applied to a Superdex ${ }^{\text {TM }} 75$ 10/30 column (GE Healthcare, Little Chalfont, UK) equilibrated with the same buffer complemented with $0.2 \mathrm{M}$ of sodium chloride.

Protein identity was further confirmed by liquid chromatography-mass spectrometry (LC/MS) analysis using MicroTOF-QII (Bruker Daltonics, Billerica, MA, USA) equipment after trypsin gel digestion of the band representing GtCel12A.

\section{2. $C D$}

Aiming to determine the secondary structure content of GtCel12A, samples of the purified protein at a concentration of $150 \mu \mathrm{g} \cdot \mathrm{mL}^{-1}$ in $50 \mathrm{mM}$ sodium citrate buffer $\mathrm{pH} 3.0$ were placed in a quartz cuvette of $0.1 \mathrm{~cm}$ path length for $\mathrm{CD}$ measurements at $10{ }^{\circ} \mathrm{C}$.

The GtCel12A CD spectra were assessed by far-UV CD spectroscopy using a J-815 Jasco spectropolarimeter equipped with a Jasco Peltier PTC 423S/15 (JASCO, Tokyo, Japan) temperature 
control unit. The CD spectra were collected using a wavelength range of 260-195 nm with a scanning speed of $100 \mathrm{~nm} \cdot \mathrm{min}^{-1}$, a spectral bandwidth of $1 \mathrm{~nm}$ and a response time of 0.5 millisecond. The protein signal was obtained by subtracting buffer spectrum from the sample spectrum and represented the average of 8 accumulations. The $\mathrm{CD}$ spectra deconvolution was performed with the program CONTINLL from the package CDPro [26].

A thermal denaturation assay was also performed by measuring the ellipticity changes induced by a temperature increase from 10 to $90{ }^{\circ} \mathrm{C}$, in steps of $10{ }^{\circ} \mathrm{C}$ in the beginning of the experiment and steps of $5{ }^{\circ} \mathrm{C}$ above $50{ }^{\circ} \mathrm{C}$ and wavelength ranging from 240 to $200 \mathrm{~nm}$. The apparent $T_{\mathrm{m}}$ was further calculated from the experimental CD sigmoid curve using the Boltzmann function.

\subsection{Native PAGE}

Protein samples of GtCel12A in $50 \mathrm{mM}$ sodium citrate buffer, $\mathrm{pH} 3.0$ in two different concentrations, 0.5 and $1.0 \mathrm{mg} \cdot \mathrm{mL}^{-1}$ were analyzed by native PAGE. The experiment was performed in the equipment Phast System (Amersham Biosciences, Little Chalfont, UK), using a nondenaturing gel, the Phast Gel 8-25 with acrylamide gradient of 8\%-25\% (GE, Little Chalfont, UK). Proteins with high $M_{\mathrm{w}} \mathrm{s}$ were used as markers, such as bovine thyroglobulin (669 kDa), horse ferritin (445 kDa), catalase $(232 \mathrm{kDa})$, aldolase $(140 \mathrm{kDa})$ and bovine serum albumin $(66 \mathrm{kDa})$. The gel was stained with Coomassie Blue in an aqueous solution of $25 \%$ methanol and $5 \%$ acetic acid.

\subsection{SAXS Studies}

SAXS data for GtCel12A at concentrations of 1 and $6 \mathrm{mg} \cdot \mathrm{mL}^{-1}$ were collected on the SAXS2 beamline at the Brazilian Synchrotron Light Laboratory (LNLS/CNPEM-ABTLuS, Campinas, Brazil). The radiation wavelength was set to $1.48 \AA$ and a $165 \mathrm{~mm}$ MAR-165 CCD detector (Rayonix, Evanston, IL, USA) was used to record the scattering patterns. The sample-to-detector distance was set to $1474 \mathrm{~mm}$ to give a scattering vector-range from 0.01 to $0.22 \AA^{-1}$, where $q$ is the magnitude of the $q$-vector defined by $q=4 \pi \sin \theta / \lambda$ ( $2 \theta$ is the scattering angle). Protein samples were prepared in $50 \mathrm{mM}$ sodium citrate buffer $\mathrm{pH}$ 3.0. The integration of SAXS patterns were performed using Fit2D software [27] and the curves were scaled by protein concentration. $R_{g}$ was approximated using two independent procedures: by Guinier equation [28] and by indirect Fourier transform method using GNOM program [29]. The $\mathrm{p}(\mathrm{r})$ was also evaluated by GNOM and $D_{\max }$ was obtained. Protein $M_{\mathrm{w}}$ was calculated using the procedure implemented on SAXSmoW web tool [14]. DAMs were calculated from the experimental SAXS data using ab initio procedure implemented in Gasbor program [16]. Several runs of ab initio shape determination with different starting conditions led to self-consistent results as judged by the structural similarity of the output models, yielding nearly identical scattering patterns and fitting statistics. CRYSOL program [30] was used to calculate the simulated scattering curve from the homologous AaXEG [18], a GH12 member, which showed 44\% of sequence identity with GtCel12A. The evaluation of $R_{\mathrm{g}}$ and $D_{\max }$ was also performed with the same program.

Finally, the global compactness of GtCel12A in $\mathrm{pH} 3.0$ was analyzed in terms of the Kratky plot [20], using a thermal variation assay with temperatures ranging from 20 to $65^{\circ} \mathrm{C}$ in steps of $10{ }^{\circ} \mathrm{C}$ in the beginning of the experiment and steps of $5{ }^{\circ} \mathrm{C}$ above $50{ }^{\circ} \mathrm{C}$. 


\section{Conclusions}

The search for enzymes with improved activity and stability has been the goal of many studies in the context of enzymatic hydrolysis of biomass. Such improvement of enzymes, as well as the discovery of new enzymes, is key to reducing the costs of converting cellulose to ethanol. In this study, we obtained important data about the structure of GtCel12A in solution, such as its oligomeric state, dimensions, shape and information about molecule flexibility. The influence of temperature was investigated and confirmed that this is an important parameter to be considered for the structural stability of GtCel12A and other enzymes applied to second generation biofuel production.

\section{Acknowledgments}

We thank Andressa P. A. Pinto from the Biophysics group of the University of Sao Paulo for technical support during CD measurements. We also thank the Brazilian Synchrotron Light Laboratory (LNLS/CNPEM-ABTLuS, Campinas, Brazil) for the use of SAXS2 beamline. This work was supported by Brazilian agency: Fundação de Amparo à Pesquisa do Estado de São Paulo (Fapesp), via grant number \#2009/08233-9.

\section{Author Contributions}

Lis S. Miotto, Caio V dos Reis and Mario O. Neto performed the experimental work. Lis S. Miotto also contributed to writing the paper. Igor Polikarpov was responsible for supervision and revision of the paper.

\section{Conflicts of Interest}

The authors declare no conflict of interest.

\section{References}

1. Goodell, B. Brown-rot fungal degradation of wood: Our evolving view. In Wood Deterioration and Preservation: Advances in Our Changing World; Goodell, B., Nicholas, D.D., Schultz, T.P., Eds.; American Chemical Society Publication: Washington, DC, USA, 2003; pp. 97-118.

2. Eriksson, K.E.L.; Blanchette, R.A. Microbial and Enzymatic Degradation of Wood and Wood Components; Springer-Verlag: Berlin, Germany, 1990.

3. Kerem, Z.; Jensen, K.A.; Hammel, K.E. Biodegradative mechanism of the brown rot basidiomycete Gloeophyllum trabeum: Evidence for an extracellular hydroquinone-driven fenton reaction. FEBS Lett. 1999, 446, 49-54.

4. Lynd, L.R.; Weimer, P.J.; van Zyl, W.H.; Pretorius, I.S. Microbial cellulose utilization: Fundamentals and biotechnology. Microbiol. Mol. Biol. Rev. 2002, 66, 506-577.

5. Gilkes, N.R.; Henrissat, B.; Kilburn, D.G.; Miller, R.C.; Warren, R.A. Domains in microbial beta-1,4-glycanases: Sequence conservation, function, and enzyme families. Microbiol. Rev. 1991, $55,303-315$. 
6. Palonen, H. Role of Lignin in the Enzymatic Hydrolysis of Lignocellulose; VTT Biotechnology: Espoo, Finland, 2004.

7. Henrissat, B. A classification of glycosyl hydrolases based on amino acid sequence similarities. Biochem. J. 1991, 280, 309-316.

8. Cantarel, B.L.; Coutinho, P.M.; Rancurel, C.; Bernard, T.; Lombard, V.; Henrissat, B. The Carbohydrate-Active EnZymes database (CAZy): An expert resource for Glycogenomics. Nucleic Acids Res. 2009, 37, D233-D238.

9. Bauer, M.W.; Driskill, L.E.; Callen, W.; Snead, M.A.; Mathur, E.J.; Kelly, R.M. An endoglucanase, EglA, from the hyperthermophilic archaeon Pyrococcus furiosus hydrolyzes beta-1,4 bonds in mixed-linkage (1-->3),(1-->4)-beta-D-glucans and cellulose. J. Bacteriol. 1999, $181,284-290$.

10. Bok, J.D.; Yernool, D.A.; Eveleigh, D.E. Purification, characterization, and molecular analysis of thermostable cellulases CelA and CelB from Thermotoga neapolitana. Appl. Environ. Microbiol. 1998, 64, 4774-4781.

11. Wicher, K.B.; Abou-Hachem, M.; Halldórsdóttir, S.; Thorbjarnadóttir, S.H.; Eggertsson, G.; Hreggvidsson, G.O.; Nordberg Karlsson, E.; Holst, O. Deletion of a cytotoxic, $N$-terminal putatitive signal peptide results in a significant increase in production yields in Escherichia coli and improved specific activity of Cel12A from Rhodothermus marinus. Appl. Microbiol. Biotechnol. 2001, 55, 578-584.

12. Ding, S.; Adney, W.S.; Vinzant, T.B.; Decker, S.R.; Baker, J.O.; Thomas, S.R.; Himmel, M.E. Glycoside hydrolase gene cluster of Acidothermus cellulolyticus. In Applications of enzymes to lignocellulosics; Mansfield, S.D., Saddler, J.N., Eds.; American Chemical Society: Washington, DC, USA, 2003; pp. 332-360.

13. Sandgren, M.; Gualfetti, P.J.; Shaw, A.; Gross, L.S.; Saldajeno, M.; Day, A.G.; Jones, T.A.; Mitchinson, C. Comparison of family 12 glycoside hydrolases and recruited substitutions important for thermal stability. Protein Sci. 2003, 12, 848-860.

14. Fischer, H.; de Oliveira Neto, M.; Napolitano, H.B.; Polikarpov, I.; Craievich, A.F. Determination of the molecular weight of proteins in solution from a single small-angle X-ray scattering measurement on a relative scale. J. Appl. Crystallogr. 2010, 43, 101-109.

15. ExPASy-ProtParam tool. Available online: http://web.expasy.org/protparam/ (accessed on 2 April 2013).

16. Svergun, D.I.; Petoukhov, M.V.; Koch, M.H. Determination of domain structure of proteins from X-ray solution scattering. Biophys. J. 2001, 80, 2946-2953.

17. Kozin, M.B.; Svergun, D.I. Automated matching of high- and low-resolution structural models. J. Appl. Crystallogr. 2001, 34, 33-41.

18. Yoshizawa, T.; Shimizu, T.; Hirano, H.; Sato, M.; Hashimoto, H. Structural basis for inhibition of xyloglucan-specific endo- $\beta$-1,4-glucanase (XEG) by XEG-protein inhibitor. J. Biol. Chem. 2012, 287, 18710-18716.

19. Glatter, O.; Kratky, O. Small Angle X-ray Scattering; Academic Press: London, UK, 1982.

20. Doniach, S.; Bascle, J.; Garel, T.; Orland, H. Partially folded states of proteins: Characterization by X-ray scattering. J. Mol. Biol. 1995, 254, 960-967. 
21. Rambo, R.P.; Tainer, J.A. Characterizing flexible and intrinsically unstructured biological macromolecules by SAS using the Porod-Debye law. Biopolymers 2011, 95, 559-571.

22. Storms, R.; Zheng, Y.; Li, H.; Sillaots, S.; Martinez-Perez, A.; Tsang, A. Plasmid vectors for protein production, gene expression and molecular manipulations in Aspergillus niger. Plasmid 2005, 53, 191-204.

23. Aslanidis, C.; de Jong, P. Ligation-independent cloning of PCR products (LIC-PCR). Nucleic Acids Res. 1990, 18, 6069-6074.

24. Debets, A.J.M.; Bos, C.J. Isolation of small protoplasts from Aspergillus niger. Fungal Genet. Newsl. 1986, 33, 24.

25. Käfer, E. Meiotic and mitotic recombination in Aspergillus and its chromosomal aberrations. Adv. Genet. 1977, 19, 33-131.

26. Chen, Y.H.; Yang, J.T. A new approach to the calculation of secondary structures of globular proteins by optical rotatory dispersion and circular dichroism. Biochem. Biophys. Res. Commun. 1971, 44, 1285-1291.

27. Hammersley, A.P. FIT2D: An Introduction and Overview; ESRF Internal Report, ESRF97HA02T; European Synchrotron Radiation Facility: Grenoble, France, 1997.

28. Guinier, A.; Fournet, G. Small-Angle Scattering of X-rays; John Wiley and Sons: New York, NY, USA, 1955; pp. 1-268.

29. Svergun, D.I. Determination of the regularization parameter in indirect-transform methods using percentual criteria. J. Appl. Crystallogr. 1992, 25, 495-503.

30. Svergun, D.I. Restoring low resolution structure of biological macromolecules from solution scattering using simulated annealing. Biophys. J. 1999, 76, 2879-2886.

(C) 2014 by the authors; licensee MDPI, Basel, Switzerland. This article is an open access article distributed under the terms and conditions of the Creative Commons Attribution license (http://creativecommons.org/licenses/by/3.0/). 